\section{The effect of appointment rescheduling on monitoring interval and patient attendance in the glaucoma outpatient clinic}

\begin{abstract}
Aim To assess the effect of appointment rescheduling on monitoring intervals and patient attendance in the glaucoma clinic. Methods A retrospective review of $\mathbf{1 0 0}$ consecutive patients was conducted. Patients were categorised into groups based on risk of progressive visual loss. The monitoring interval requested by the clinician was compared with the interval recommended by national guidelines. One hundred consecutive patients who had had their appointment rescheduled were also examined to assess the effect of appointment rescheduling on the actual monitoring interval. Patient nonattendance rates and attendances at the emergency department were also examined. Results After excluding patients with secondary glaucoma, glaucoma suspects, and those with short-term factors affecting monitoring interval, 54 patients with chronic open-angle glaucoma (COAG) were included. Forty-eight $(89 \%)$ of patients had a monitoring interval requested in accordance with national guidelines ( 2 had longer intervals by 1-2 months and 4 had shorter time intervals). The monitoring interval was not influenced by disease severity (Kruskal-Wallis test, $P=0.16$ ), but was significantly shorter if the intraocular pressure was above target $(P<0.0001)$ or the patient showed structural or functional progression $(P<\mathbf{0 . 0 0 0 1})$. Hospital-initiated appointment rescheduling led to significant lengthening of monitoring interval. The mean difference between intended and actual monitoring interval was $\mathbf{5 . 6}$ months. Eight percent $(8 / 100)$ of patients with
\end{abstract}

A Tatham ${ }^{1}$ and I Murdoch ${ }^{1,2}$

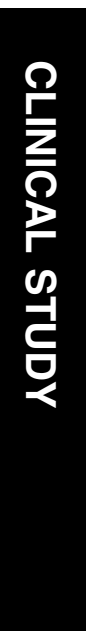

rescheduled appointments did not attend compared with $15 \%$ (39/265) without rescheduled appointments.

Conclusion Clinical staff are selecting appropriate monitoring intervals for patients with COAG; however, hospital-initiated rescheduling of appointments is a major challenge to appropriate follow-up.

Eye (2012) 26, 729-733; doi:10.1038/eye.2012.22; published online 2 March 2012

Keywords: glaucoma; clinical guidelines; outpatient

Introduction

Patients with chronic open-angle glaucoma (COAG) should be monitored according to risk of disease progression and visual loss. ${ }^{1,2}$ Examples of appropriate monitoring intervals have been outlined in guidance from organisations such as the National Institute of Clinical Excellence (NICE), ${ }^{2}$ the European Glaucoma Society, ${ }^{3}$ and the American Academy of Ophthalmology. ${ }^{4}$ If the interval between examinations is inappropriately long, patients may experience progression that might otherwise have been prevented. Delay in glaucoma patient follow-up appointment provision has emerged as a significant cause of patient safety incidents. Over a 4-year period, the United Kingdom National Patient Safety Agency (NPSA) received reports of 44 patients with glaucoma, including 13 patients with total loss of vision, who had experienced deterioration of vision attributed to delayed follow-up appointments. ${ }^{5}$ Although patients
${ }^{1}$ Moorfields Eye Hospital NHS Foundation Trust, London, UK

${ }^{2}$ Institute of Ophthalmology, London, UK

Correspondence: A Tatham, Moorfields Eye Hospital NHS Foundation Trust, 162 City Road, London EC1V 2PD, UK

Tel: $+44(0) 2072533411$; Fax: + 44 (0)20 72534696 E-mail: andrewtatham@ yahoo.co.uk

Received: 22 November 2011

Accepted in revised form: 20 January 2012 Published online: 2 March 2012 
may come to harm because of delayed appointments, too frequent monitoring is inefficient, inconvenient for the patient and relatives, and may leave insufficient capacity in clinics to review patients who are at higher risk. Reasons for inappropriate monitoring interval are likely to include an inappropriate interval requested by the clinician, insufficient clinic capacity, and patient nonattendance. Hospital-initiated rescheduling of appointments may also adversely affect the monitoring interval and encourage patient non-attendance. These factors are likely to become increasingly challenging as the increasing and ageing population creates further pressure on services. ${ }^{6}$

The aims of this study were to examine monitoring intervals in a busy glaucoma clinic and to assess the effect of appointment rescheduling on patient attendance patterns.

\section{Materials and methods}

This study was approved by the institutional audit board and followed the tenants of the Declaration of Helsinki. The initial stage of the study involved determining the proportion of patients with COAG attending the glaucoma clinic who had monitoring intervals requested in accordance with the risk of progressive visual loss. Risk of progressive visual loss was defined according to the risk classification scheme detailed in the NICE guidelines (Table 1). A retrospective review of 100 consecutive case records was performed for patients attending on one day in August 2011. Only patients with COAG were included. Patients with secondary glaucoma, suspect glaucoma, ocular hypertension, angleclosure glaucoma, and short-term factors likely to affect monitoring interval, specifically intraocular surgery or glaucoma laser surgery within 1 year, were excluded. Staff working in the clinic included glaucoma specialists, residents, and optometrists, and all were unaware that the study was being conducted that day. Patients with
COAG were categorised into one of five groups based on risk of progressive visual loss using the NICE glaucoma guidelines classification. ${ }^{2}$ Group allocation depended on whether the intraocular pressure (IOP) in the patient's worse eye was at target and whether there was evidence of progression based on optic nerve damage and/or visual field change (Table 1). The attainment of target IOP was based on whether the assessing clinician had judged the IOP to be at an acceptable level. The presence or absence of the optic nerve or visual field progression was also based on the judgement of the assessing clinician, as documented in the patient's case record, and was determined by stereoscopic optic disc examination and photographs, Heidelberg retina tomograpy, and Humphrey automated perimetry.

Patients were also classified by severity as having early, moderate, or advanced glaucoma. Early glaucoma was defined as early glaucomatous disc features with a vertical cup-to-disc ratio $\leq 0.65 \pm$ mild visual field defect; moderate glaucoma was defined as moderate glaucomatous disc features with a vertical cup-to-disc ratio of $0.7-0.85$ or moderate visual field defect not involving $10^{\circ}$ of fixation; and advanced glaucoma was defined as advanced disc damage with a vertical cup-to-disc ratio $\geq 0.9$ and/or field defect within $10^{\circ}$ of fixation. ${ }^{7}$ The monitoring interval requested by the consulting specialist was noted and this was compared with the monitoring interval recommended by the NICE guidelines.

The second stage of the study examined the effect of appointment rescheduling on the actual monitoring interval. One hundred consecutive patients who had had their current appointment rescheduled by the hospital were included. We did not include appointments rescheduled by the hospital because of the patient failing to attend their previous appointment. Appointment dates were reviewed using the clinic appointment

Table 1 Monitoring intervals for people with COAG (adapted from NICE glaucoma guidelines)

\begin{tabular}{|c|c|c|c|c|c|c|}
\hline \multicolumn{3}{|c|}{ Clinical assessment } & \multirow{2}{*}{$\begin{array}{l}\text { Number } \\
\text { of patients }\end{array}$} & \multicolumn{2}{|c|}{ NICE recommended monitoring interval (months) } & \multirow{2}{*}{$\begin{array}{l}\text { Monitoring interval requested } \\
\text { by clinician (months) }\end{array}$} \\
\hline $\begin{array}{l}\text { Group } \\
\text { number }\end{array}$ & $\begin{array}{l}\text { IOP at } \\
\text { target }^{\mathrm{a}}\end{array}$ & Progression $^{\mathrm{b}}$ & & IOP alone & $\begin{array}{l}\text { IOP, optic nerve } \\
\text { head, visual field }\end{array}$ & \\
\hline 1 & Yes & $\mathrm{No}^{\mathrm{c}}$ & 23 & Not applicable & $6-12$ & $\begin{array}{l}\text { Mean } 6.9 \pm 2.3 \\
\text { Range } 4-12\end{array}$ \\
\hline 2 & Yes & Yes & 2 & $1-4$ & $2-6$ & Mean $2.0 \pm 0$ \\
\hline 3 & Yes & Uncertain & 5 & Not applicable & $2-6$ & $\begin{array}{l}\text { Mean } 4.8 \pm 1.8 \\
\text { Range } 4-8\end{array}$ \\
\hline 4 & No & $\mathrm{No}^{\mathrm{c}}$ & 7 & $1-4$ & $6-12$ & $\begin{array}{l}\text { Mean } 3.0 \pm 2 \\
\text { Range } 2-6\end{array}$ \\
\hline 5 & No & Yes/uncertain & 17 & $1-2$ & $2-6$ & $\begin{array}{l}\text { Mean } 2.0 \pm 0.8 \\
\text { Range } 1 \text { week to } 6 \text { months }\end{array}$ \\
\hline
\end{tabular}

${ }^{\text {a IOP }}$ at or below target.

${ }^{b}$ Progression, increased optic nerve damage and/or visual field change confirmed by repeated test where clinically appropriate.

'No, not detected or not assessed if IOP check only following treatment change. 
software and by verifying these with the patient. The actual monitoring interval was compared with the monitoring interval requested by the clinician at last review. Patient non-attendance rates and attendances at the accident and emergency department in the interval between visits were also examined.

\section{Results}

One hundred consecutive patients were identified from those attending the glaucoma clinic. Fourteen patients were excluded because of short-term surgical factors likely to affect monitoring interval. In the 12 months preceding the study visit, 10 patients had undergone Baerveldt tube or trabeculectomy surgery, 3 patients had needed cataract extraction, and one patient had been treated with cyclodiode laser. Thirty-two other patients were excluded as they had secondary glaucoma (3 patients), angleclosure glaucoma (8 patients), or did not have glaucoma (12 glaucoma suspects, 8 ocular hypertensives, and one patient with a visual field defect not due to glaucoma). Therefore, a total of 54 patients with COAG were included for further analysis. The characteristics of these patients are summarised in Table 2.

There were 15 patients with early glaucoma, 10 patients with moderate glaucoma, and 29 patients with advanced glaucoma. At the study visit 59\% (32/54) patients had no change in treatment, 35\% (19/54) had their glaucoma medication altered, and $6 \%(3 / 54)$ were scheduled for

Table 2 Characteristics of patients with chronic open angle glaucoma included in analysis

\begin{tabular}{|c|c|c|}
\hline $\begin{array}{l}\text { Patient } \\
\text { characteristics }\end{array}$ & Worse eye & Better eye \\
\hline \multicolumn{3}{|c|}{ VF mean deviation at study visit } \\
\hline Mean & $-10.9 \pm 8.6$ & $-5.0 \pm 5.1$ \\
\hline Max & 0.3 & 1.2 \\
\hline \multirow[t]{2}{*}{ Min } & -33.9 & -4.2 \\
\hline & $\begin{array}{l}6 \text { eyes visual acuity too } \\
\text { poor for visual field }\end{array}$ & $\begin{array}{l}3 \text { eyes visual acuity too } \\
\text { poor for visual field }\end{array}$ \\
\hline \multicolumn{3}{|c|}{ Severity of glaucoma (number of patients) } \\
\hline None & 0 & 5 \\
\hline Early & 15 & 23 \\
\hline Moderate & 10 & 11 \\
\hline Severe & 29 & 15 \\
\hline \multicolumn{3}{|c|}{ Baseline IOP (mm Hg) } \\
\hline Mean & $25.0 \pm 7.7$ & $23.3 \pm 5.8$ \\
\hline Max & 45 & 43 \\
\hline Min & 0 (1 phthisical) & 15 \\
\hline \multicolumn{3}{|c|}{ IOP at study visit $(\mathrm{mm} \mathrm{Hg})$} \\
\hline Mean & $16.8 \pm 5.2$ & $16.3 \pm 4.1$ \\
\hline Max & 32 & 30 \\
\hline Min & 0 & 9 \\
\hline
\end{tabular}

glaucoma surgery. The mean monitoring interval requested was $4.6 \pm 2.9$ months (range 1 week to 12 months).

Patients were allocated into five groups based on the NICE glaucoma guidelines' classification of risk of progressive visual loss. There were 23 patients in group 1, 2 patients in group 2, 5 patients in group 3, 7 patients in group 4, and 17 patients in group 5 (Table 1). In all, $48 / 54(89 \%)$ of patients had a monitoring interval requested in accordance with that recommended by NICE. Two patients had a monitoring interval longer than recommended ( 1 and 2 months longer, respectively) and four had a shorter interval than recommended (three patients 2 months shorter and one patient 3 weeks shorter).

The monitoring interval requested was not influenced by disease severity (Kruskal-Wallis test, $P=0.16$ ). The mean requested monitoring intervals were $5.6 \pm 3.2$ months for patients with early glaucoma, $3.5 \pm 3.0$ months for patients with moderate glaucoma, and $4.4 \pm 2.5$ months for those with advanced glaucoma. In contrast, patients deemed to be progressing were more likely to have monitoring requested at an earlier interval than those who were not progressing. If the patient had evidence of structural or functional progression the mean monitoring interval requested was $1.9 \pm 0.7$ months, if progression was uncertain the mean interval was $3 \pm 1.8$ months, and if the patient had no sign of progression the mean interval was $6.1 \pm 2.7$ months (Kruskal-Wallis test, $\left.\chi^{2}=20.41, P<0.0001\right)$. Similarly, if the IOP was at target the mean monitoring interval was $6.2 \pm 2.6$ months, if target IOP was uncertain the mean interval was $4.7 \pm 1.2$ months, and if the IOP was not at target the mean interval was $2.1 \pm 1.0$ months (Kruskal-Wallis test, $\chi^{2}=22.50, P<0.0001$ ) (Figure 1).

For the second phase of the study we reviewed 100 consecutive patients whose appointment had been rescheduled by the hospital. Three clinic days were necessary to achieve this number. Over the three days a total of 365 appointments had been booked, consisting of 60 new and 305 follow-up patients. One hundred out of $365(27 \%)$ of the appointments had been previously rescheduled by the hospital. Hospital-initiated appointment rescheduling led to significant lengthening of monitoring interval. The mean interval intended until the next appointment was $7.3 \pm 2.9$ months, whereas the actual interval was $12.7 \pm 4.1$ months. The mean difference between the intended monitoring and actual monitoring interval was $5.6 \pm 2.9$ months, representing a $42.6 \pm 14.5 \%$ increase in duration between appointments. Thirteen percent of the 365 appointment slots were wasted because of patient non-attendance. Eight out of $100(8 \%)$ of patients with rescheduled appointments did not attend, compared with 39/265 (15\%) without rescheduled appointments. Six percent of 


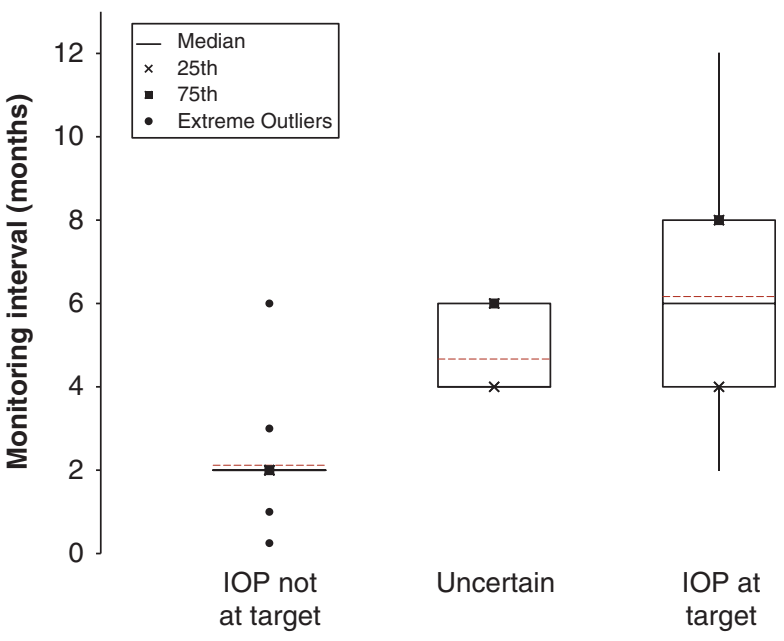

Figure 1 Monitoring interval requested and IOP control.

patients with rescheduled appointments attended the ophthalmology accident and emergency clinic in the interval between appointments. Of those that attended the rescheduled appointment, 7/92 (8\%) were deemed to have progressed during the interval between appointments, therefore suffering harm potentially attributable to the delay in follow-up.

\section{Discussion}

The results of this study indicate that clinical staff are selecting appropriate monitoring intervals for patients with COAG. Nine out of ten patients had their next appointment scheduled within an interval recommended by national guidelines. Of the six patients for whom the requested monitoring interval was not within NICE guidelines, four patients had shorter intervals requested and two patients had intervals 1 and 2 months longer than recommended. The monitoring interval selected depended largely on whether the patient's IOP was at target and whether the patient was showing signs of structural or functional progression. In contrast, the severity of glaucoma did not influence the monitoring interval.

These results also demonstrate that hospital-initiated rescheduling of appointments is a major challenge to the appropriate monitoring of patients with glaucoma. In this sample, over one-quarter of patients attending the glaucoma clinic had their initial appointment rescheduled. Consequently, although the attending ophthalmologist may have scheduled a review appointment within the period recommended by national guidelines, the eventual appointment was often much later.

The large number of rescheduled appointments and the difficulties of rescheduling within an appropriate time interval reflect the high demands on ophthalmology and glaucoma services. Patients may be rescheduled when additional appointments are required, often at short notice, for new patients, postoperative patients, and those deemed at high risk of progression. Rescheduling may lead to patient dissatisfaction and potentially exposes the patient to harm. In this series, $8 \%$ of patients had progression that may have been preventable if their appointment had not been delayed. Appointment rescheduling has been highlighted as a major safety issue by the NPSA, ${ }^{5}$ and was emphasised in a recent editorial examining patient safety issues in ophthalmology. ${ }^{8}$

Patient safety should be of prime concern for all healthcare providers and forms a key component of highquality care. Quality of care is coming under increased scrutiny and is now linked to financial reimbursement schemes. For example, the NHS Commissioning for Quality and Innovation payment framework makes a proportion of provider income conditional on the achievement of quality improvement goals. ${ }^{9}$ Quality will be measured against standards such as those published by $\mathrm{NICE}^{5}$ and the Royal College of Ophthalmologists. ${ }^{10}$ Indicators of quality included in the college standards include having a named clinical lead for glaucoma, reviewing patients with glaucoma in dedicated clinics, and auditing glaucoma-related visual impairment certification rates. The standards also recommend that appointments occur within $15 \%$ of their intended follow-up period; therefore patients with a rescheduled 6-month appointment should be seen within 7 months. In this series, only $2 \%$ of patients had their appointment rescheduled within $15 \%$ of the intended interval time.

Quality Statements 6 and 8 of the NICE Glaucoma Quality Standards are particularly relevant to this study. Quality Statement 6 states that people diagnosed with glaucoma should be monitored at intervals appropriate to their risk of progressive visual loss. ${ }^{1}$ Quality Statement 8 states that people with glaucoma have access to timely follow-up appointments and investigations, that sufficient capacity is in place to provide this service, and that systems are developed to identify people needing clinical priority if appointments are cancelled, delayed, or missed. ${ }^{1}$

Previous research in other specialties has suggested that rescheduling appointments encourages nonattendance. ${ }^{11}$ In the ophthalmology outpatient clinic, King et $a l^{12}$ reported an overall non-attendance rate of $12.6 \%$ over 12 months. Review patients were 1.09 times more likely to non-attend than new patients. Other factors associated with an increased risk of non-attendance include young age, male sex, and suburban address. ${ }^{11,13}$ In this study rescheduling appointments did not increase patient non-attendance, perhaps as patients with rescheduled appointments were anxious to be seen after a long interval between 
visits. Indeed patients may seek review elsewhere and rescheduling may adversely affect other services. We found that $6 \%$ of patients with rescheduled appointments attended the ophthalmology accident and emergency department in the period between reviews in the glaucoma clinic.

We acknowledge that this study has weaknesses, particularly as this was a single-centre investigation and our experiences may not be reflected elsewhere. Furthermore, decisions regarding monitoring interval are complex and may not be accurately captured using the five groups of the NICE guidelines. For example, patients recently diagnosed may need more frequent examinations to establish a baseline and rule out rapid progression. ${ }^{14}$ It has recently been shown that three visual field examinations per year are required to detect a 4-dB change in mean deviation over 2 years with adequate statistical power. ${ }^{14}$ The frequency of examination should also be increased if there is a higher perceived risk of functional visual loss, high IOP, visual field progression, or factors such as pseudoexfoliation, increased age, or family history of glaucoma blindness. ${ }^{14}$ Also, of paramount importance, but beyond the scope of this study, is the quality of examination at each visit.

Despite treatment and monitoring, patients with glaucoma still become blind and rescheduling of appointments may be a factor in some. ${ }^{15}$ Approaches to reduce hospital-initiated rescheduling might include reducing wasted appointments due to patient nonattendance, freeing capacity through referral refinement, increasing capacity through extra staff and clinic time, and developing new ways of working, such as sharedcare and virtual clinics. What is clear is that it is important to address the problem of appointment rescheduling in order to improve patient satisfaction and prevent incidents of avoidable patient harm.

\section{Summary}

\section{What was known before}

- Patients with chronic open-angle glaucoma require lifelong monitoring and treatment in order to minimise the risk of disease progression. Examples of appropriate monitoring intervals have been outlined in guidelines such as those from the National Institute of Clinical Excellence. Appointment delays for patients with glaucoma are a major patient safety issue.

What this study adds

- Most patients have a monitoring interval selected within the duration recommended by national guidelines, however, hospital-initiated rescheduling of appointments is a major challenge to appropriate monitoring. Monitoring intervals are shorter for patients with an intraocular pressure not at target or for those showing signs of structural or functional progression. The severity of glaucoma did not influence the monitoring interval.

\section{Conflict of interest}

The authors declare no conflict of interest.

\section{Acknowledgements}

We are grateful for the work of Valerie Giddings in facilitating data collection.

\section{References}

1 Department of Health. NICE glaucoma quality standard, March 2011. http://www.nice.org.uk/guidance/ qualitystandards/qualitystandards.jsp.

2 National Institute of Clinical Excellence (NICE) guidance CG85. Glaucoma: diagnosis and management of chronic open angle glaucoma and ocular hypertension, April 2009. http://guidance.nice.org.uk/CG85.

3 European Glaucoma Society. Terminology and Guidelines for Glaucoma, 3rd edn. European Glaucoma Society: Safona, Italy, 2008.

4 American Academy of Ophthalmology. Preferred Practice Pattern. Primary Open-Angle Glaucoma. American Academy of Ophthalmology: San Francisco, CA, 2010, p25.

5 National Patient Safety Agency. Preventing delay to follow-up for patients with glaucoma. Rapid Response Report 2009; NPSA/2009/RRR004. http://www.nrls.npsa. nhs.uk/resources $/$ ?entryid $45=61908 \& p=2$.

6 Mitchell P, Smith W, Attebo K, Healey PR. Prevalence of open-angle glaucoma in Australia. The Blue Mountains Eye Study. Ophthalmology 1996; 103(10): 1661-1669.

7 Damji KF, Behki R, Wang L. Canadian perspectives in glaucoma management: setting target intraocular pressure range. Can J Ophthalmol 2003; 38(3): 189-197.

8 Kelly SP. Update on patient safety. Br J Ophthalmol 2011; 95: 1183-1184.

9 Department of Health. Using the Commissioning for Quality and Innovation (CQUIN) payment framework. December 2008. http://www.dh.gov.uk/en/ Publicationsandstatistics/Publications/ PublicationsPolicyAndGuidance/DH_091443.

10 The Royal College of Ophthalmologists Quality Standard Development Group. Quality Standards for Glaucoma Services. August 2011. http://www.rcophth.ac.uk/ page.asp? section $=444$ \{sectionsign $\}$ ionTitle $=$ Quality + Standards.

11 Frankel S, Farrow A, West R. Non-attendance or noninvitation? A case-control study of failed outpatient appointments. BMJ 1989; 298(6684): 1343-1345.

12 King A, David D, Jones HS, O'Brien C. Factors affecting non-attendance in an ophthalmic outpatient department. J R Soc Med 1995; 88(2): 88-90.

13 Leese AM, Wilson JA, Murray JA. A survey of the non-attendance rate at the ENT clinic of a district general hospital. Clin Otolaryngol Allied Sci 1986; 11(1): 37-40.

14 Chauhan BC, Garway-Heath DF, Goni FJ, Rossetti L, Bengtsson B, Viswanathan AC et al. Practical recommendations for measuring rates of visual field change in glaucoma. Br J Ophthalmol 2008; 92(4): 569-573.

15 Chen PP. Blindness in patients with treated open-angle glaucoma. Ophthalmology 2003; 110(4): 726-733. 